\title{
Specific Solutions of a Class of Second Order Difference Equation with Boundary Conditions
}

\author{
Y. Ouyang \\ School of Mathematics and Statistics \\ Hechi University \\ Guangxi, P. R. China
}

\begin{abstract}
Difference equation is a kind of important tool to study the rule of natural phenomena. In this paper, we discuss several specific solutions of a class of second order difference equation with boundary conditions.
\end{abstract}

Keywords-difference equation; second order; boundary condition; specific solution

\section{INTRODUCTION}

Difference equations is a kind of important tool to study the rule of natural phenomena, such as, physical problems arising in a wide variety of applications. Cheng and Cho [3] investigated the following second order difference equations

$$
\Delta^{2} x(k-1)+p(k) x(k)=0,
$$

where $p(k)$ is a real valued function defined on a set of the natural numbers.

Motivated by the results given in [1, 2, 3, 4, 5], in this paper, we discuss speciffc solutions of the following second order difference equations for $k \in\{1,2, \cdots N\}$,

$$
\Delta^{2} x(k-1)+p(k) x(k)=0,
$$

satisfying

$$
x(0)=0, x(N+1)=0 \text {, }
$$

or

$$
x(0)+\sigma x(1)=0, x(N+1)+\lambda x(N)=0,
$$

or

$x(0)+\sigma x(1)=0, x(2 m)+\lambda x(2 m)=0, x(4 m+1)+\theta x(4 m)=0$.

\section{MAIN RESULTS}

Throughout this paper, let $\mathrm{n}, \mathrm{m}$ be natural numbers,

$$
I_{n, m}=\{n, n+1, \cdots, m\}
$$

Proposition 1. Let $N=2 m+1$,

$$
p(k)=\left\{\begin{array}{cc}
0, & k \in I_{0, m,}, \\
2 /(m+1), & k=m+1, \\
0, & k \in I_{m+2, N},
\end{array}\right.
$$

and

$$
x(k)=\left\{\begin{array}{cc}
k, & k \in I_{0, m+1}, \\
2 m-k+2, & k \in I_{m+1, N+1} .
\end{array}\right.
$$

Then (3) is the specific solution of second order difference equation (1).

Proof. From (2) and (3), we have

$$
\begin{aligned}
& \frac{\Delta^{2} x(m)}{x(m+1)} \\
& =\frac{x(m+2)-2 x(m+1)+x(m)}{x(m+1)} \\
& =\frac{m-2(m+1)+m}{m+1} \\
& =\frac{-2}{m+1}=-p(m+1)
\end{aligned}
$$$$
\text { Since } \quad \Delta^{2} x(k-1) / x(k)=0=-p(k)
$$

$k \in I_{1, m} \cup k \in I_{m+2, N}$, (3) is a specific solution of (1) with $x(0)=0, x(N+1)=0$.

Proposition 2. Let $N=2 m$,

$$
p(k)=\left\{\begin{array}{cc}
0, & k \in I_{0, m,} \\
(2 m+1) / m(m+1), & k=m+1, \\
0, & k \in I_{m+2, N}
\end{array}\right.
$$

and

$$
x(k)=\left\{\begin{array}{cc}
k, & k \in I_{0, m+1}, \\
(m+1)(2 m-k+1) / m, & k \in I_{m+1, N+1} .
\end{array}\right.
$$

Then (6) is the specific solution of (1).

Proof. From (5) and (6), we obtain that

$$
\begin{aligned}
& \frac{\Delta^{2} x(m)}{x(m+1)} \\
& =\frac{x(m+2)-2 x(m+1)+x(m)}{x(m+1)} \\
& =\frac{\left(m^{2}-1\right) / m-2(m+1)+m}{m+1} \\
& =\frac{m^{2}-1-2 m(m+1)+m^{2}}{m(m+1)} \\
& =-\frac{2 m+1}{m+1}=-p(m+1) .
\end{aligned}
$$


Since

$$
\Delta^{2} x(k-1) / x(k)=0=-p(k)
$$

for

$k \in I_{1, m} \cup k \in I_{m+2, N}$, (6) is a specific solution of (1) such that

$$
x(0)=0, x(N+1)=0 .
$$

Proposition 3. Let $N=2 m+1$,

$$
p(k)=\left\{\begin{array}{cc}
0, & k \in I_{0, m,}, \\
\frac{N+1+N \lambda+N \sigma+2 m \lambda \sigma}{(m+1+m \sigma)(m+1+m \lambda)}, & k=m+1, \\
0, & k \in I_{m+2, N},
\end{array}\right.
$$

and

$$
x(k)=\left\{\begin{array}{cc}
1+(1+\sigma)(k-1), & k \in I_{0, m+1,} \\
\frac{(m+1+m \sigma)[2 m+1+1 /(1+\lambda)-k]}{m+1 /(1+\lambda)}, & k \in I_{m+1, N+1 .}
\end{array}\right.
$$

Then (9) is the specific solution of (1) with

$$
x(0)+\sigma x(1)=0, x(N+1)=\lambda x(N)=0 .
$$

Proof. From (8) and (9), we conclude

$$
\begin{aligned}
& \frac{\Delta^{2} x(m)}{x(m+1)} \\
= & \frac{x(m+2)-2 x(m+1)+x(m)}{x(m+1)} \\
= & \frac{x(m+2)-x(m+1)-(x(m+1)-x(m))}{x(m+1)} \\
= & \frac{1+\sigma}{m+1+m \sigma}+\frac{m+1+m \sigma}{(m+1 /(1+\lambda))(m+1+m \sigma)} \\
= & \frac{1+\sigma}{m+1+m \sigma}+\frac{1+\lambda}{m+1+m \lambda} \\
= & \frac{(1+\sigma)(m+1+m \lambda)+(1+\lambda)(m+1+m \sigma)}{(m+1+m \sigma)(m+1+m \lambda)} \\
= & \frac{2 m+2+(2 m+1) \lambda+(2 m+1) \sigma+2 m \lambda \sigma}{(m+1+m \sigma)(m+1+m \lambda)} \\
= & \frac{N+1+N \lambda+N \sigma+2 m \lambda \sigma}{(m+1+m \sigma)(m+1+m \lambda)}=-p(m+1) \\
& \operatorname{Since} \\
& \Delta^{2} x(k-1) / x(k)=0=-p(k) \text { for } k \in I_{1, m} \bigcup k \in I_{m+2, N},
\end{aligned}
$$

(9) is a specific solution of (1) with

$$
x(0)+\sigma x(1)=0 . \quad x(N+1)+\lambda x(N)=0 .
$$

Proposition 4. Let $N=2 m$,

$$
p(k)=\left\{\begin{array}{cc}
0, & k \in I_{0, m-1,} \\
\frac{N+1+N \lambda+N \sigma+(N-1) \lambda \sigma}{(m+(m-1) \sigma)(m+1+m \lambda)} & k=m \\
0, & k \in I_{m+1, N}
\end{array}\right.
$$

and

$$
x(k)=\left\{\begin{array}{cl}
1+(1+\sigma)(k-1) & k \in I_{0, m,}, \\
\frac{(m(1+\sigma)-\sigma)(2 m+1 /(1+\lambda)-k)}{m+1 /(1+\lambda)}, & k \in I_{m, N+1,}
\end{array}\right.
$$

Then (12) is the solution of (1) with $x(0)+\sigma x(1)=0, x(N+1)+\lambda x(N)=0$

Proof. Using (11) and (12), we have

$$
\begin{aligned}
- & \frac{\Delta^{2} x(m-1)}{x(m)} \\
= & -\frac{x(m+1)-2 x(m)+(m-1)}{x(m)} \\
= & -\frac{x(m+1)-x(m)-(x(m)-x(m-1))}{x(m)} \\
= & \frac{m(1+\sigma)-\sigma}{(m+1 /(1+\lambda))(m(1+\sigma)-\sigma)}+\frac{1+\sigma}{m(1+\sigma)-\sigma} \\
= & \frac{1+\sigma}{m+(m-1) \sigma}+\frac{1+\lambda}{m+1+m \lambda} \\
= & \frac{(1+\sigma)(m+1+m \lambda)+(1+\lambda)(m+(m-1) \sigma)}{(m+(m-1) \sigma)(m+1+m \lambda)} \\
= & \frac{2 m+1+2 m \lambda+2 m \sigma+(2 m-1) \lambda \sigma}{(m+(m-1) \sigma)(m+1 m \lambda)} \\
= & p(m), \\
& \Delta^{2} x(k-1) / x(k)=0=-p(k)
\end{aligned}
$$

$k \in I_{1, m} \cup k \in I_{m+2, N}$, (12) is a specific solution of (1) with $x(0)+\sigma x(1)=0, x(N+1)+\lambda x(N)=0$.

Proposition 5. Let

$$
p(k)=\left\{\begin{array}{cc}
0, & k \in I_{0, m-1,} \\
\frac{2 m+1+2 m \lambda+2 m \sigma+(2 m-1) \lambda \sigma}{(m+(m+1) \sigma)(m+1+m \lambda)}, & k=m \\
0, & k \in I_{m+1,3 m-1,} \\
\frac{2 m+2 m \lambda+2 m \theta+2 m \lambda \theta+\lambda-\theta}{(m+m \theta+1)(m+m \lambda-1)}, & k=3 m, \\
0, & k \in I_{3 m+1,4 m},
\end{array}\right.
$$

and

$$
x(k)=\left\{\begin{array}{cc}
1+(1+\sigma)(k-1), & k \in I_{0, m}, \\
\frac{(m(1+\sigma)-\sigma)(2 m+1 /(1+\lambda)-k)}{m+1 /(1+\lambda)}, & k \in I_{m, 3 m}, \\
\frac{(m(1+\sigma)-\sigma)(1 /(1+\lambda)-m)}{(m+1 /(1+\lambda))(m+1 /(1+\theta))} \times\left(4 m+\frac{1}{1+\theta}-k\right), & k \in I_{3 m, 4 m+1 .} .
\end{array}\right.
$$

Then (15) is the solution of (1) with $x(0)+\sigma x(1)=0, x(2 m+1)+\lambda x(2 m)=0, x(4 m+1)+\theta x(4 m)=0$

Proof. Using (14) and (15), we have

$-\frac{\Delta^{2} x(m-1)}{x(m)}=\frac{2 m+1+2 m \lambda+2 m \sigma+(2 m-1) \lambda \sigma}{(m+(m-1) \sigma)(m+1+m \lambda)}=p(m)$,

$-\frac{\Delta^{2} x(3 m-1)}{x(3 m)}$

$=-\frac{x(3 m+1)-2 x(3 m)+x(3 m-1)}{x(3 m)}$ 


$$
\begin{aligned}
& =-\frac{x(3 m+1)-x(3 m)-(x(3 m)-x(3 m-1))}{x(3 m)} \\
& =\frac{1+\theta}{m+m \theta+1}+\frac{1+\lambda}{m+m \lambda-1} \\
& =\frac{(1+\theta)(m+m \lambda-1)+(1+\lambda)(m+m \theta+1)}{(m+m \theta+1)(m+m \lambda-1)} \\
& =\frac{2 m+2 m \lambda+2 m \theta+2 m \lambda \theta+\lambda-\theta}{(m+m \theta+1)(m+m \lambda-1)} \\
& =p(3 m),
\end{aligned}
$$

Since

$$
\Delta^{2} x(k-1) / x(k)=0=-p(k)
$$

for $k \in I_{1, m-1} \bigcup I_{m+1,3 m-1} \bigcup k \in I_{3 m+1,4 m}$, (15) is a specific solution of (1) with

$$
x(0)+\sigma x(1)=0, x(2 m+1)+\lambda x(2 m)=0, x(4 m+1)+\theta x(4 m)=0
$$

\section{SUMMARY}

Difference equation is a kind of important tool to study the rule of natural phenomena. In this paper, we discuss several specific solutions of a class of second order difference equation with boundary conditions.

\section{ACKNOWLEDGEMENTS}

This research was supported by Scientific Research Foundation of the Education Department of Guangxi Autonomous Region of China (No. 201010LX473).

\section{REFERENCES}

[1] E. J. Moulton, A theorem in difference equations on the alternation of nodes of linearly independent solutions. Annals of Mathematics, 13, pp. 137-139, 1911-1912.

[2] E. Makai, On a monotonic property of certain sturm-liouville functions. Acta Mathematica Acaiemiae Scientiarum Hungaricae, 3, pp. 165-172, 1952.

[3] S. S Cheng and A. M. Cho, Convexity of nodes of discrete sturmliouville functions. Hokkaido Mathematical Journal, 11, pp. 8-14,1982.

[4] S. S Cheng, A discrete analogue of the inequality of lyapunov. Hokkaido Mathematical Journal, 12, pp. 105-112, 1983.

[5] B. D. Pachpatte, On lyapunov-type inequalities for certain higher order differential equations. Journal of mathematical analysis and applications, 195, pp. 527-536, 1995. 